\title{
Intelligent Anti-Grouping in Real-Time Strategy Games
}

\author{
Nicola Beume, Tobias Hein, Boris Naujoks, Nico Piatkowski, Mike Preuss, Simon Wessing
}

\begin{abstract}
Assembling suitable groups of fighting units to combat incoming enemy groups is a tactical necessity in realtime strategy (RTS) games. Furthermore it heavily influences future strategic decisions like unit building. Here, we demonstrate how to efficiently (offline) solve the problem of finding matches for the current enemy group(s) based on self-organizing maps (SOMs), powered by a simple evolutionary algorithm. The concept is implemented and thoroughly experimentally investigated in the RTS game Glest. We show that the offline learning is reliable and can be sped up considerably by employing a very simple substitute objective function instead of game simulations, making it a nearly universal, simple, and transparent technique.
\end{abstract}

\section{INTRODUCTION}

To be fair, and thus interesting, strategy games have to be thoroughly balanced. The easiest way to achieve this is by providing every player with identical options. The available unit types are especially interesting for the strategy. Usually, the quality of a strategy heavily depends on the strategy of the current opponent. With the change of the opponent's strategy, the optimal strategy will change. In such a situation of intransitive superiority (see fig. 1), a global optimum is not present due to the fact that there is no partial ordering of the strategies. So, there is no point in trying to globally optimize a strategy.

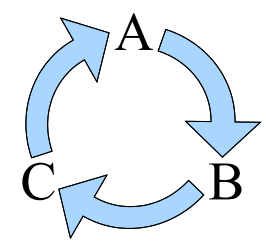

Fig. 1. With three strategies $A, B$ and $C$, we may have a dependency that strategy $B$ beats strategy $A(A \prec B), B \prec C$ and $C \prec A$ (also called "Rock, Paper, Scissors"-situation after the homonymous children's game).

Instead, a good game AI shall adapt to the strategy of the (human) opponent, leading to better performance and to a more believable AI at the same time, as this is what human players also do. One important part of the strategy is unit group matching. It has two direct tactical uses, and a strategic one.

- Tactical attack: If an enemy base or unit group shall be attacked by the AI, and some information about the defenders is available, it is favorable to bring enemy units that perform well against them (e.g. $A$ beats $B$ in the scenario of fig. 1).

All authors are with the Chair of Algorithm Engineering, Computational Intelligence Group, Faculty of Computer Science, Technische Universität Dortmund, Germany. E-mail: Nicola.Beume@tu-dortmund.de, Tobias.Hein@tu-dortmund.de, Boris.Naujoks@tu-dortmund.de, Nico.Piatkowski@tudortmund.de, Mike.Preuss@tu-dortmund.de, Simon.Wessing@ tu-dortmund.de
- Tactical defense: In case the AI is attacked, possibly by multiple enemy groups, it makes sense to send the available units against the enemy units they can beat first, before they are annihilated by units they cannot tackle.

- Strategic build: On a strategic layer, the unit build process - and thus also the exploration of the tech tree, including buildings - shall take into account information about the favored unit types of the adversary.

Whereas for the latter use, data must be gathered and accumulated on a longer time scale, the tactical decisions have to be taken ad hoc, or with a very small time lag. In most RTS games, the 'fog of war' hides attacking enemy units until they are already very near to own units or buildings, so that an AI may not use valuable seconds thinking over possible answers. One way to circumvent the need for fast decisions would be to cheat, so that the AI gets the information earlier. But, as we show here, this is usually not necessary, as appropriate solutions to the anti-group selection problem can be learned offline and then employed by a simple lookup procedure. Furthermore, the learning process itself is quite fast as our technique does not even need game simulations but performs well with a properly chosen very simple substitute objective function. In the following, we concentrate on the tactical defense scenario. However, the two others may be treated analogously.

We address the anti-group selection problem by incorporating a self-organizing map as a filter into the AI. The major advantage of this approach is transparency: Resulting maps - as e.g. opposed to standard artificial neural networks (ANNs) - allow for a meaningful visualization, the maps can be understood by humans. They are not black boxes what may facilitate the application of this technique also by nonCI experts.

In this work, we use Glest, an open source RTS, for investigating and demonstrating the suitability of our approach. Glest has been used as a test bench for several CI methods before [1]. The game provides a configurable intransitive superiority relation of the different unit types by employing a damage multiplier table. It is used to compute the actual damage inflicted by an attack of a specific unit type onto another by modifying it according to the armor type the defending unit is equipped with. The default multipliers for the damage are given in table I.

The following sections first introduce Glest and its strategic options in $\S \mathrm{II}$, SOMs and their use in our context in $\S$ III, a substitute objective function to be used instead of simulations of the game in $\S \mathrm{IV}$ and related research in $\oint \mathrm{V}$. The experiments are described in $\S \mathrm{VI}$ and conclusions are drawn in $\S$ VII. 
TABLE I

DAMAGE MULTIPLIERS OF ATTACK TYPES AGAINST ARMOR TYPES IN THE TECH FACTIONS.

\begin{tabular}{|c|c|c|c|c|}
\hline & Leather & Plate & Stone & Wood \\
\hline Arrow & 1.5 & 0.5 & 0.3 & 1.0 \\
Sword & 1.0 & 1.0 & 0.5 & 1.0 \\
Fire & 1.0 & 0.8 & 0.5 & 1.0 \\
\hline
\end{tabular}

\section{GLEST}

Glest is an open source RTS game published by Figueroa et al. [2]. While this work is based on version 2.0.1, Glest version 3 has been released with an improved gameplay and balancing. Therefore some of the observations shown here might not be relevant to the new version.

The game is settled in a fantasy medieval world. The players can choose to control either a magic or a tech faction. Both factions feature several unit types, buildings and enhancements. While the game also has other aspects like resource harvesting and building, this work only deals with battle situations, and only with combat units of the tech faction. Basic unit types are archers and swordmen, both armored with leather. Armored swordmen are slower than the two mentioned, but possess a plate armor. Horsemen are equally well armored and much faster. The technician, a weak unit, is prerequisite for all following units. The catapult is slow, but deals a high damage. The battle-machine is very versatile, being the only unit that has a short- and a long-range weapon. At last, there are two flying units: The airship, a kind of bomber, and the ornithopter. All units that are constructed by technicians feature a wooden armor. Archers and battle-machines are the only ground units that can attack flying units. The units take up different resources and usually become available in the mentioned order during the game. This complex setting provides the base for a variety of strategies.

\section{Self-Organizing MAPS For Unit SELECTION}

The Self-Organizing Map (SOM) was conceived by Kohonen [3] in 1982. A SOM maps a high-dimensional data space to a low-dimensional, usually 2 -dimensional map space combining the goals of visualization and dimension reduction of complex data sets. SOMs are topology-preserving maps, i.e. while mapping the data from the input space to the map space they consider the underlying structure of the data and attempt to obtain the topological relationships. Nearby elements of the input space are also close to each other in the projected map space. Consequently a SOM creates a map of regions of similarity by grouping similar data.

The SOM consists of a set of nodes, which are arranged in a discrete lattice defining the topology of the map. Different topologies exist such as rectangular or hexagonal arrangements of nodes. In our case we defined a toroid-shaped lattice by connecting opposite edges of the map. Each node $i$ of the lattice is associated with a randomly initialized reference or weight vector $w_{i}=\left(\omega_{i 1}, \omega_{i 2}, \ldots, \omega_{i n}\right)^{T} \in R^{n}$. The dimension $n$ of the weight vectors correspond to the dimension of the input vector. The input vector $x=\left(\epsilon_{1}, \epsilon_{2}, \ldots, \epsilon_{n}\right)^{T}$ specifies the distribution of unit types over an opponent's army. Likewise, the weight vectors $w_{i}$ represent a mix of the defending player's unit types.

Basically the learning algorithm conforms to the original algorithm devised by Kohonen [3]. In each iteration $t$ an input vector $x$ is randomly drawn from the data set using a desired probability distribution over the input space. While traversing the SOM's nodes the distance between the input vector $x$ and each node's weight vector $w_{i}$ is calculated. The applied distance measure can be an arbitrary function that takes two objects as input and returns a scalar value like the Euclidean distance. The node with the shortest distance to the input vector, thus the node that matches the input vector best is called the best-matching unit (BMU). The BMU's weight vector is manipulated to approximate the input vector even further. Nodes within a defined radius $r$ around the BMU are being adapted as well. The influence $\ell$ of the adaption is defined by a Gaussian curve having its center at the BMU and making it fall off with increasing distance to the BMU. The neighborhood size shrinks with increasing iterations. The learning process will finally terminate after a certain number of iterations is reached.

Operation of the SOM just works by looking up the BMU in the same way as during training, but without the step of manipulating the nodes. The found BMU (and its neighborhood) deliver information that may then be processed further.

\section{Substitute Objective Function}

Usually, SOMs are used in conjunction with a (weighted) Euclidean distance. A specialty of our work is the development of a non-standard distance measure for this purpose. It is designed to relate to suitability instead of similarity. Consider for example the following situation: During the game, the AI gathers the information that the opponent is about to attack with a certain group of units $E$. A part of the problem to find the optimal defending group is the question: How successful would be a certain group $U$ that is facing the attackers? Of course, this could be answered by a simulation of the upcoming battle in the game. Such a function is implemented by starting the battle between the two groups in the game. The simulation ends when one party is defeated or after 5,000 game cycles elapsed. While $p_{E}$ and $p_{U}$ denote the fractions of surviving units from the attacking and defending groups, the outcome is measured as $\left(p_{E}-p_{U}\right) \rightarrow \min$ ! at the end of the game. The optimum of this function is -1.0 , which means that all attacking units have been eliminated, while all defending units survived. There likely is not enough time to run a simulation during the game. So, to keep the computational costs low, we developed a substitute objective function $f(E, U)$ based on our knowledge of the properties of the game's units. The function returns a score of a group $U$ against a hostile group $E$. In our example situation, $E$ is the attacking group and $U$ is the AI's defending group. In our setup, $U$ is retrieved from the AI's memory, the SOM. 
A unit can have several attack skills. Every skill $s$ is evaluated with a score $c_{s}$ against the set of attackable units $E_{s}$ in the opponent's group $E . d\left(t_{s}, r_{e}\right)$ is the damage multiplier of attack-type $t_{s}$ against armor-type $r_{e}$ of hostile unit $e$.

$$
c_{s}=\sum_{e \in E_{s}} d\left(t_{s}, r_{e}\right)
$$

Every unit $u$ in group $U$ is awarded a score $c_{u}$ for its most useful attack skill only. $c_{u}$ is calculated as

$$
c_{u}=\max \left\{c_{s, 1}, \ldots, c_{s, n}\right\} .
$$

A group's score $f(E, U)$ then is the sum of all its units' scores $c_{u}$. To account for the mutual strengths and weaknesses, the final objective is $(f(U, E)-f(E, U)) \rightarrow$ min!.

Obviously, the function does neither take costs like time and resources, nor features like speed, hitpoints and attackstrength into account. Finding an optimal group under these additional constraints would be a highly multi-criteria problem that is not covered by our approach. So, the objective function only considers aspects that characterize the relative strength of units. Preliminary experiments showed that else only the most expensive units would benefit.

As described in section III, it is necessary to adapt defending groups to the attacking ones in the learning process. While this is a trivial task with Euclidean distance, we need to optimize the substitute objective function. Here, a $(1+1)$ EA carries out the optimization of the defender's mix of units. An introduction to evolutionary algorithms (EA) is given by Beyer and Schwefel in [4]. Incorporating an EA into a SOM has already been proposed by Kohonen [3], [5].

The influence $\ell$ of adaptation is controlled by the number of objective function evaluations. Every time the BMU is found during the training, the EA is being run for $\lfloor\ell \cdot 10\rfloor$ function evaluations. $\ell$ is 1.0 for the BMU and falls off with increasing distance. Mutation is carried out on the real number representation of the groups, by shifting a certain amount between two randomly drawn unit types. The amount that is being shifted is determined by a normal distributed random variable with mean 0.0 and variance 0.05 . The mutation-strength can be seen as analogy to the learning rate often used with a Euclidean distance.

\section{RELATED RESEARCH}

Some attempts have been made to produce RTS players that are capable of adaptive behavior. Louis et al. [6], [7] combined EAs with memories to produce strategies online in the game. While only trained offline in this paper, our SOM could serve this purpose, too. In their setup the EA performs the exploration of new plans, while the memory delivers previously successful plans to speed up the search. Like in the SOM, solutions are retrieved from the memory with a distance measure. Louis et al. only implemented a distance measure based on the Hamming distance of the solution's genotypes. Detecting the opponent's grouping behavior is a subproblem of opponent modeling, which has been addressed by Spronck et al. [8] as well as knowledge acquisition in RTS games in general [9].
TABLE II

DAMAGE MULTIPLIERS OF ATTACK TYPES AGAINST ARMOR TYPES IN THE BALANCED FACTIONS.

\begin{tabular}{|c|c|c|c|}
\hline & Armor 1 & Armor 2 & Armor 3 \\
\hline Attack 1 & 0.5 & 5.0 & 0.5 \\
Attack 2 & 0.5 & 0.5 & 5.0 \\
Attack 3 & 5.0 & 0.5 & 0.5 \\
\hline
\end{tabular}

\section{EXPERIMENTAL EVALUATION}

We divide the experimental investigation of the suggested technique into three different experiments. An initial proofof-principle test shall demonstrate its general viability. Using an artificial setting that clearly deviates from standard Gle st, it is easy to test if the desired output (in terms of a recommended unit group) is indeed attained. To support our assumption that SOM training by means of a simple substitute objective function instead of simulations is reasonable, we then investigate the correlation between these two data sources. In a third experiment, we apply our technique to the anti-grouping problem in standard Glest, also investigating the proper parametrization and the parameter robustness of the SOM training process.

The reports on the received results adhere to the suggestion by Preuss [10]. They are thus subdivided into seven parts, namely research question, preexperimental planning, task, setup, results/visualization, observations and discussion. This division is inspired from experimentation in the natural sciences and was first suggested (for EA) for experimenting in the framework of sequential parameter optimization of Bartz-Beielstein [11]. It demands the author to think about the tackled questions and the employed means before the experiment is carried out and to report the results in a structured, easily accessible way.

Experiment 1: Proof-of-principle; does the SOM generate the 'right' results in a simplified scenario?

Preexperimental planning: According to the intransitive superiority principle, we designed a simplified scenario (balanced factions) with only three unit types employing the damage multipliers given in tab. II. In-game experimentation confirmed that this leads to the desired behavior. For each of the three unit types, there is one favored counterpart performing best.

Task: By applying a full factorial design over the SOM parameters, a good SOM configuration shall be determined, accompanied by a parameter effect estimation. A parameter is only considered as important if the result set for one level significantly $(p<0.05)$ differs from the set for the other level in a Wilcoxon rank sum test (also U-test), likewise for the two-parameter interactions. For a well configured SOM, we require an average BMU fraction of at least $90 \%$, compared to the optimal answer (homogeneous test cases with only one unit type).

Setup: We perform a full factorial design over extreme parameter values for the number of nodes on each axis 
of the SOM (dimension), the number of learning steps (iterations), the allowed unit number (unit), the number of learning patterns (always 3 in the homogeneous case), and the attacker group type, according to tab. III. Note that high unit numbers primarily increase the accuracy of the SOM, as it only uses fractions internally. For each configuration, the SOM is repeatedly trained 10 times and the resulting substitute objective function value is averaged over all (3) homogeneous and all (3) 2-unit equally shared mixed attacking groups. The BMU test is performed over four configurations. The largest SOM (dimension 16) is repeatedly (5 times) trained for 1000 iterations with 30 units and 100 mixed patterns and also homogeneous attacker groups, respectively. The smallest SOM (dimension 2) is trained under the same conditions, but only for 100 iterations and 10 units. The BMU fraction is computed from the averaged fraction of 'right' answers (archers for swordmen, swordmen for horsemen, and horsemen for archers) over the 3 homogeneous test patterns.

TABLE III

PARAMETER VALUES FOR THE FULL FACTORIAL DESIGN

$\begin{array}{lrrrrr}\text { Parameter } & \text { dim. } & \text { iterations } & \text { units } & \text { patterns } & \text { attacker } \\ \text { Low value } & 2 & 100 & 10 & 100 & \text { homogeneous } \\ \text { High value } & 16 & 1000 & 30 & 1000 & \text { mixed }\end{array}$

Results/Visualization: Main effects and 2-factor interactions are estimated via the Design of Experiments (DoE) standard procedure. We show all main effects in fig. 2 and only the significant interactions in fig. 3. The BMU detection frequencies are given in tab. IV.

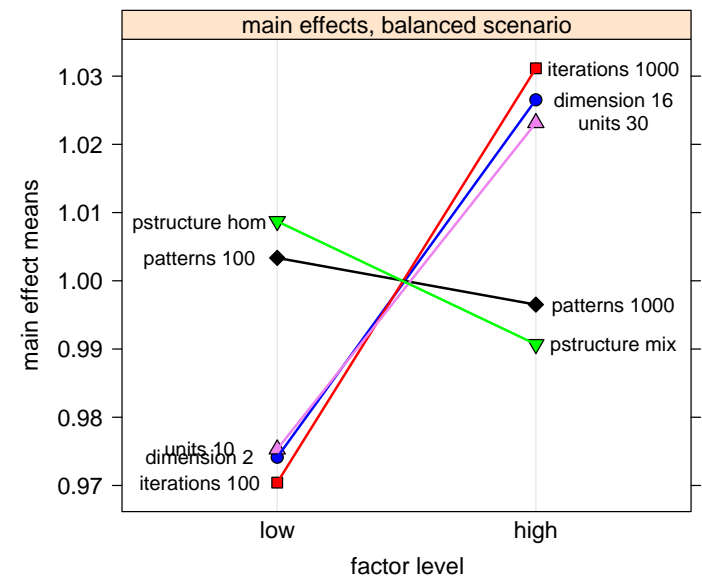

Fig. 2. Main effect estimations of parameters for the balanced scenario. All effects except the number of patterns have a significant effect.

Observations: It is no surprise that increasing the SOM size and the numbers of iterations and units leads to better performance. However, the number of patterns seems to play no role at all (or 100 is already sufficient), and strangely enough, training with only homogeneous patterns is better than with mixed patterns. The interactions behave as ex-

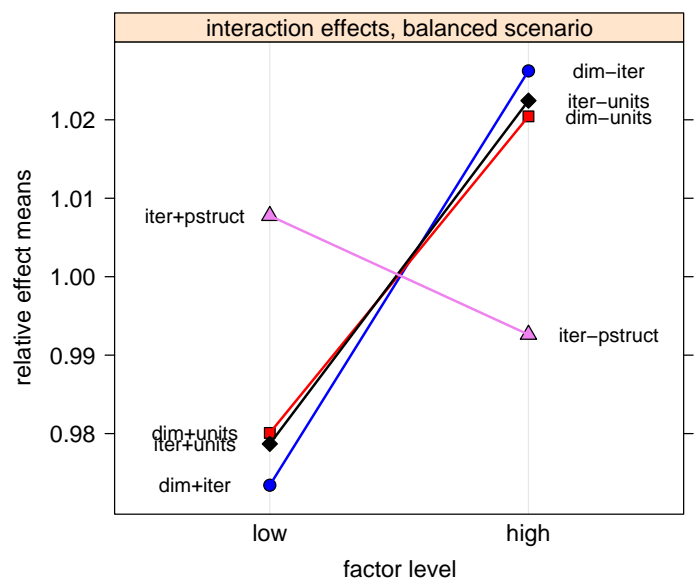

Fig. 3. Only the significant interaction effects of parameters for the balanced scenario.

TABLE IV

BMU DETECTION FREQUENCY FOR EXTREMELY CONFIGURED SOMS ON BALANCED SCENARIO

$\begin{array}{lrrrrrr}\text { Dim. } & \text { iter. } & \text { units } & \text { patterns } & \text { attacker } & \text { average } & \text { std. } \\ 2 & 100 & 10 & (3) & \text { homogeneous } & 84.09 \% & 1.968 \% \\ 2 & 100 & 10 & 100 & \text { mixed } & 84.68 \% & 7.685 \% \\ 16 & 1000 & 30 & (3) & \text { homogeneous } & 98.97 \% & 0.080 \% \\ 16 & 1000 & 30 & 1000 & \text { mixed } & 99.02 \% & 0.251 \%\end{array}$

pected, all combinations of the important factors also produce strong interactions. Overall, the effects are not very strong (about 6\% maximum difference between best and worst). The BMU detection frequencies are all relatively high, even for the small SOMs with less training iterations. However, here the variance is quite large, being much smaller for large SOMs with more training iterations.

Discussion: The balanced scenario is easy to learn, and even the small SOMs cope with it acceptably (note that a SOM size of $2 \times 2$ is minimal as we have 3 unit types). We can only speculate why the performance with homogeneous test patterns is better than with mixed ones. A possible reason could be the reduced level of randomness: Homogeneous patterns may provide clear and stable guidelines, whether mixed patterns vary a lot. Deterministic training data seems to support learning here.

Concerning the BMU detection frequencies, the weaker results for small SOMs presumably stem from conflicts between neighboring nodes. The small SOMs often learn only two of the three optima. Additionally, large SOMsinitialized randomly-also have a head start because it is much more likely that already near optimal defense unit vectors can be found in 256 trials than in 4 .

Overall, both results show that the SOM is reliable and easy to handle: It can be successfully trained with (few) homogeneous patterns, and even if the important parameters (size, iterations, and units) are set badly, performance decreases only gradually. 
Experiment 2: Does the substitute objective function properly represent simulation results on the real game?

Preexperimental planning: Different variants of the objective function as presented in $\S \mathrm{IV}$ had been tried, and of the ones that intuitively gave the impression of performing well, we chose the simplest one.

Task: We apply Kendall's rank correlation test to paired samples of simulation and substitute function results $\left(H_{0}\right.$ is that both data sources are indistinguishable) and expect a pvalue of at most $5 \%$. The computed correlation value should be positive and in the order of 0.5 .

Setup: We do not test with completely random attacking groups, as this situation would almost never occur in a concrete game (at least not while playing against humans). Instead, we generate only homogeneous or 2-type mixed attacking groups of size 10 each. The defending group is drawn randomly from all unit types, also containing 10 units (allowing randomness here makes sense as the composition of the defending group is the search space of the SOM). Much larger group sizes are infeasible for simulation as simulation time grows super linearly in unit numbers. We restrict our tests to tech level 3 (9 types; see exp. 3) which provides the largest search space for the SOM. When succeeding here, we assume that tech level 1 and 2 can also be modeled by the substitute objective function.

Results/Visualization: Table V provides correlation estimates and the associated p-values of the tests, fig. 4 gives a visualization.

TABLE V

KENDALL'S RANK CORRELATIONS AND TEST P-VALUES BETWEEN SIMULATION AND SUBSTITUTE OBJECTIVE FUNCTION

$\begin{array}{lrrr}\text { Attacker } & \text { samples } & \text { p-Value } & \text { rank correl. } \\ \text { Homogeneous groups } & 360 & <10^{-15} & 0.4234 \\ \text { Mixed groups } & 360 & <10^{-15} & 0.4238 \\ \text { Homogeneous+mixed groups } & 720 & <10^{-15} & 0.4298\end{array}$

Observations: The rank correlation values (tab. V) hardly show any difference between homogeneous and mixed samples, which is unexpected. Figure 4 largely adheres to our expectations. Still, it reveals some interesting details. At first, the simulation minimum $(-1.0)$ is nearly reached, but objective function values stay far from -100 , its minimum. Obviously, the objective function is more accurate for small values, so that simulation results can already be nearly optimal even if the optimal unit composition-according to the objective function-is not yet reached. Secondly, very good objective function values possess a very low variance in simulation values - this seems to support the previous observation. Thirdly, the -/- quadrant is very dense, meaning that there are many more good than bad (randomly drawn) defending unit compositions.

Discussion: The missing difference in rank correlation values between mixed and homogeneous samples points to another source of variation with a stronger influence. Most likely, this is the inherent randomness of the simulation

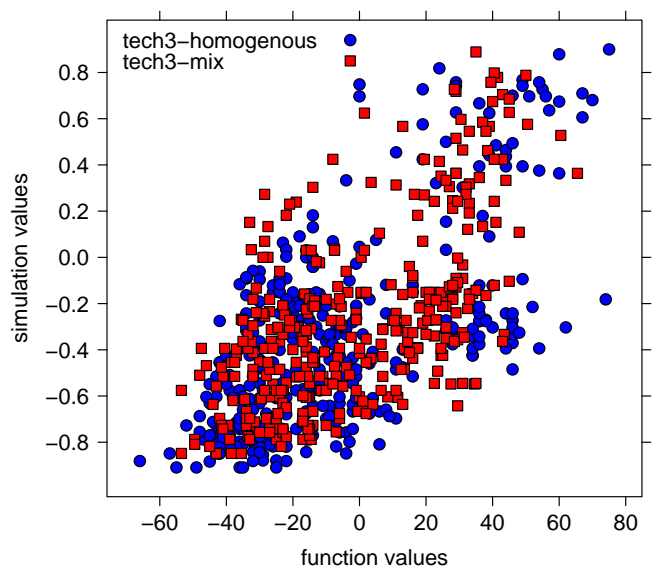

Fig. 4. Correlation plot: Simulation result over substitute objective function, 360 random samples of defenders against homogeneous and mixed attacker groups, respectively. Theoretical minima are -1.0 for the simulation and -100 for the objective function.

(damage values are partly random). We may interpret that as a quality indicator of the substitute objective function. Despite the noise in simulation data, the correlation is approximately on the required level. Applying other rank correlation metrics (e.g. Spearman) even leads to slightly higher values. The test values clearly state that the null hypothesis (no correlation) should be rejected. We deduce that the substitute objective function is sufficient for SOM training, and that due to its deterministic nature, it may be even better to use this function than to learn via simulations. Another interesting revealed fact is that there are very many good answers to a specific attacker group. Obviously, it pays off to send different troops into a combat than used by the aggressor.

Experiment 3: Do the SOMs perform well in a more complicated real game setting?

Preexperimental planning: Our first experiments with the full unit set resulted in SOMs clearly favoring air units. Thus, we partitioned the units from standard Glest into three levels. Tech-Level 1 is similar to our balanced faction from experiment 1 plus the armored swordman (but with the original damage multipliers). It consists of archer, armored swordman, horseman and swordman. Tech-Level 2 adds battle machine, catapult and the technician to Level 1 whereas the air units airship and ornithopter are added in Level 3. This is consistent with the game flow, as these are the 3 sequential phases usually experienced in Glest. All level 1 units can be built in the barracks, whereas the air units require air bases which cannot be established by the builders but only by technicians. So we do not only get three levels of difficulty as test cases for the SOM, but also 3 different types of SOMs which may each assist a Glest AI in the sequential game phases and be switched as new units become available. Task: Again, we strive for finding the significant main and interaction effects of the SOM configuration as in exp 1 . 
We aim for obtaining information about the robustness of the parameter settings in exp. 1. For being robust, we demand that the parameter recommendations from exp. 1 are suitable for exp. 3 also, or vice versa, so that at least one robust parameter setting exists. Additionally, we measure the SOMs performance by comparing its suggested defense group against the precalculated, optimal solutions obtained from total enumeration. The SOM, if properly configured, should reach at least $90 \%$ of the optimal score on average.

Setup: In order to obtain reference values for comparing the SOM performances on the substitute objective function, we enumerated the search space for each tech-level to obtain optimal defending groups for homogeneous as well as for 2-type-mixed attacking groups of size ten. For these more complicated game situations, we have no means to otherwise obtain the optimal answer of the SOMs as by optimization. For exp. 1, the scenario had been constructed in a way that the optimal answer was obvious. As the worst substitute objective function values -0 stands for a draw - are around 100 (see fig. 4, smaller is better), we compute the relative quality of a SOM answer after formula 3 . We perform 31 repeats for each SOM configuration to get an impression of the underlying distributions.

$$
q_{\text {rel }}=\frac{f_{\text {subst }}(\text { som })-100}{\text { optimum }-100}
$$

The overall setup for the SOMs is the same full factorial design as in exp. 1.

Results/Visualization: Figures 5 to 8 document the measured main effects for tech levels 1 to 3 and the 2-factor interaction effects tech level 2. For tech levels 1 and 3, only one weak interaction was detected. Table VI shows the average relative answer qualities of 2 different SOMs for tech level 3, before and after training. The SOMs are tested on all 9 homogeneous and all 362 -unit equally shared mixed attacker groups. This is also visualized in more detail in fig. 9.

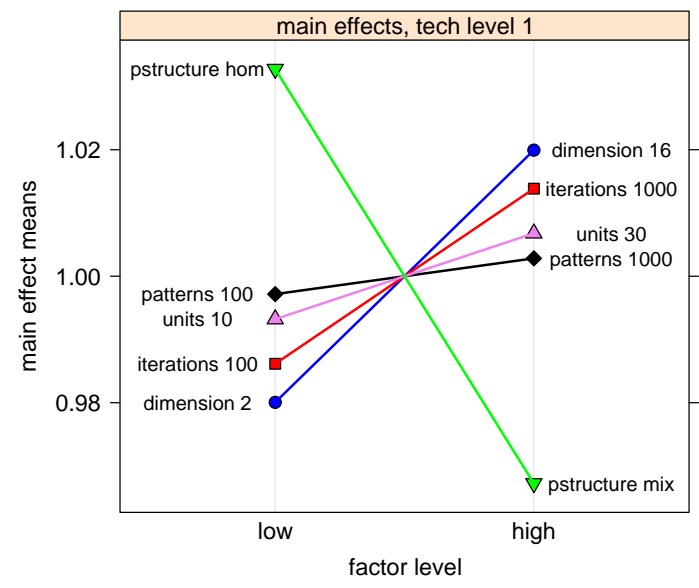

Fig. 5. Main effect estimations of parameters for the tech level 1 scenario (4 units). All effects are significant, except pattern number and unit size.

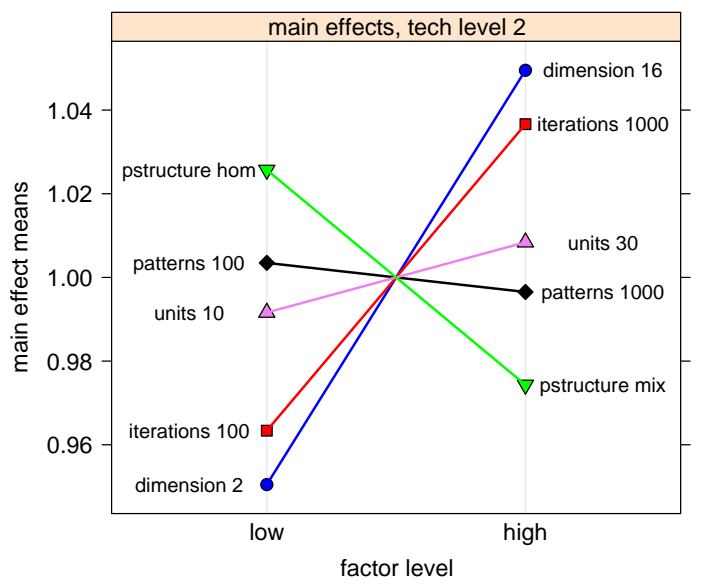

Fig. 6. Main effect estimations of parameters for the tech level 2 scenario (7 units). All except pattern number and unit size are significant, again.

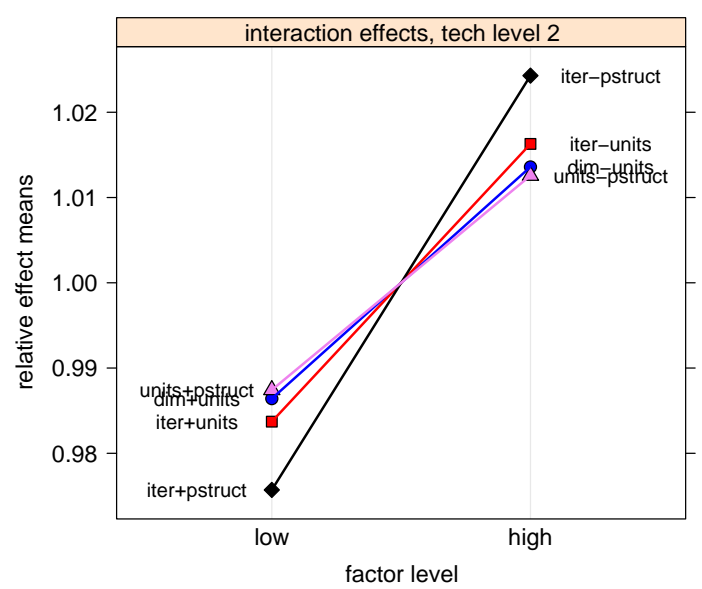

Fig. 7. Only the significant interaction effects of parameters for the tech level 2 scenario.

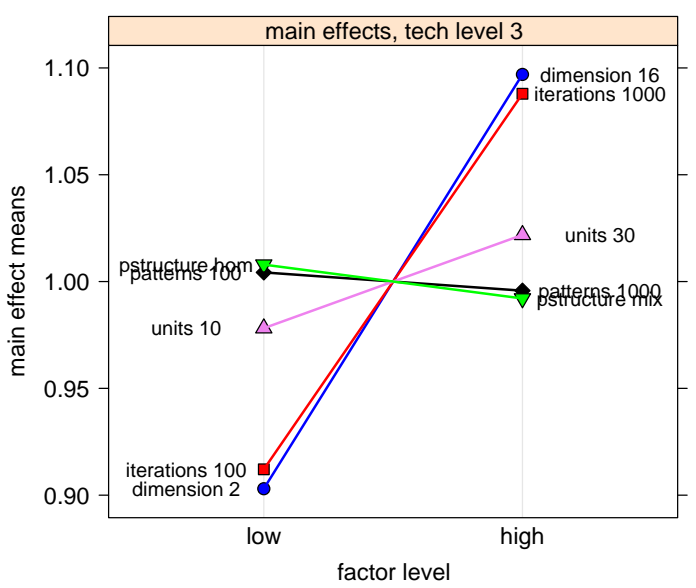

Fig. 8. Main effect estimations of parameters for the tech level 3 scenario (9 units). Here, all except pattern size and pattern structure are significant. 


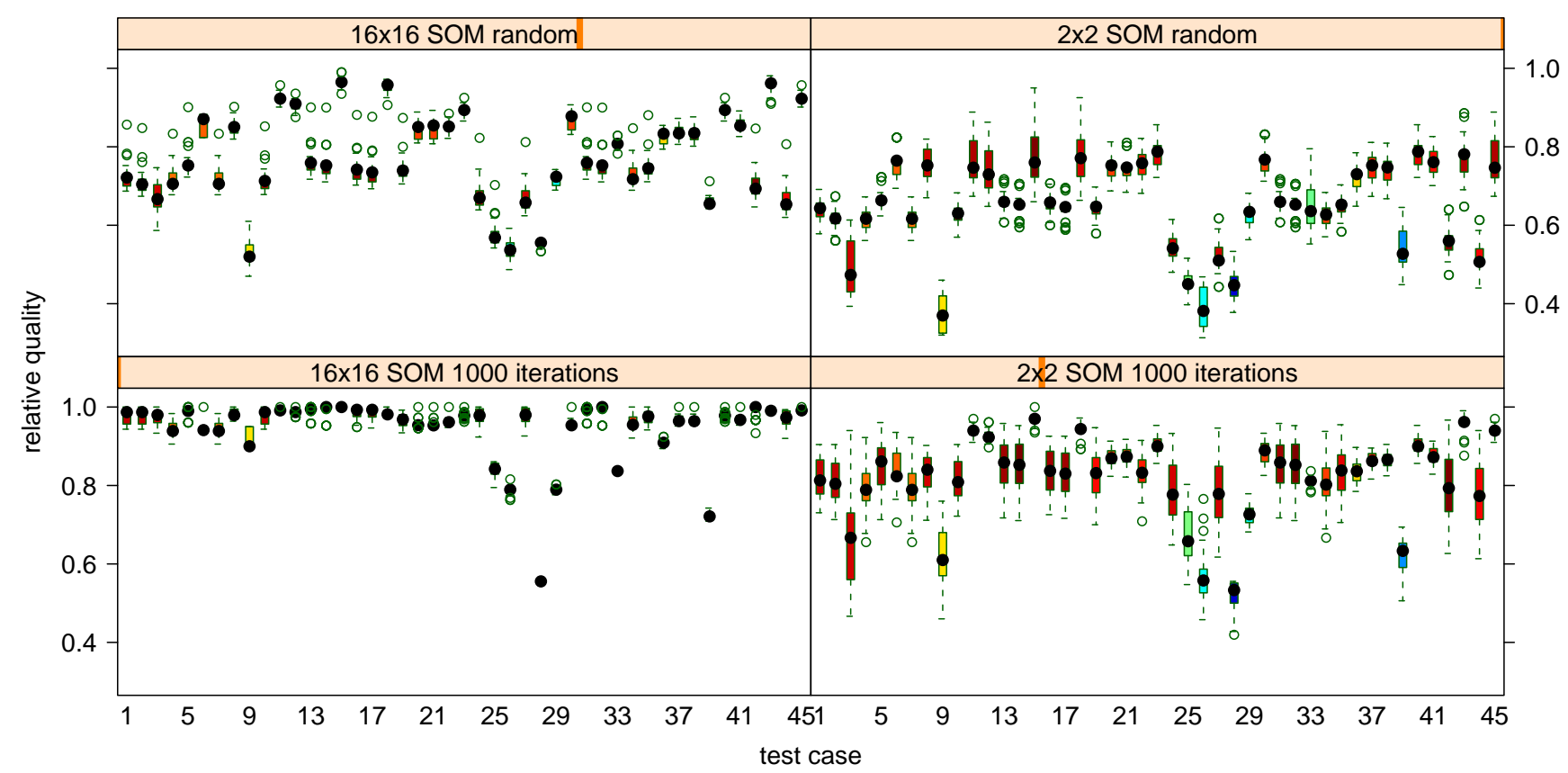

Fig. 9. Box plots of relative qualities of a large and a small SOM with and without training.
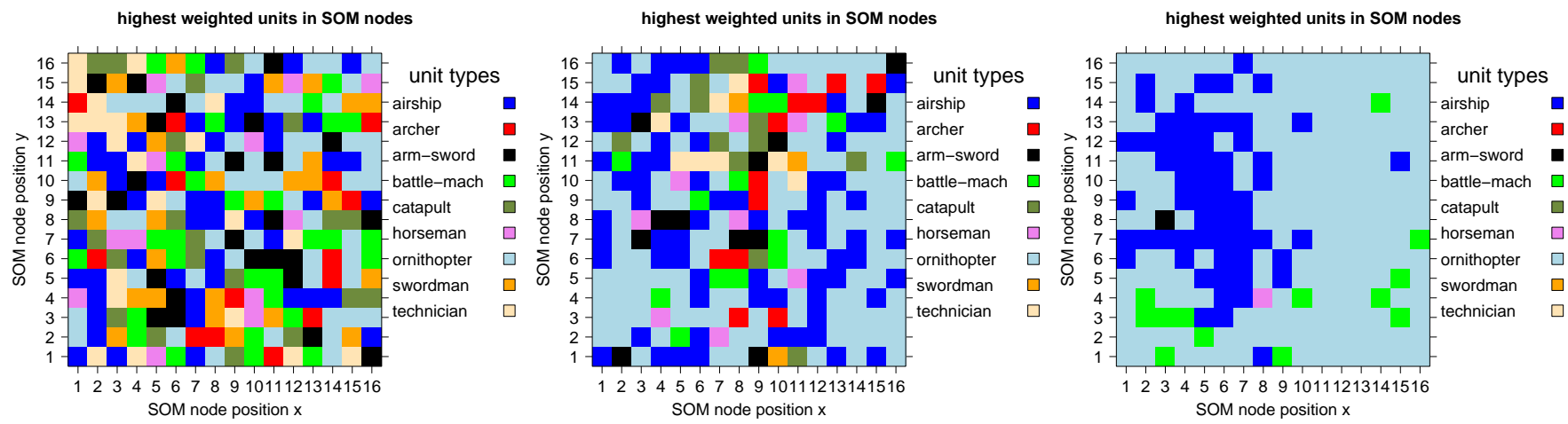

Fig. 10. Evolution of a Glest tech level $3 \mathrm{SOM}(16 \times 16,30$ units, 9 homogeneous patterns) during the training process. From left to right: After 10 , 100, and 1000 iterations, respectively. Learning ends with a clear domination of air units.

TABLE VI

RELATIVE ANSWER QUALITY OF WELL AND BADLY CONFIGURED TRAINED AND RANDOMLY ESTABLISHED SOMS (ITERATIONS=0) COMPARED TO THE OPTIMAL VALUES FOR TECH LEVEL 3

$\begin{array}{lrrrrrr}\text { Dim. } & \text { iter. } & \text { units } & \text { patterns } & \text { attacker } & \text { average } & \text { std. } \\ 16 & 1000 & 10 & (9) & \text { homogeneous } & 94.32 \% & 8.52 \% \\ 16 & 0 & 10 & (9) & \text { homogeneous } & 77.15 \% & 11.04 \% \\ 2 & 100 & 10 & (9) & \text { homogeneous } & 81.68 \% & 9.85 \% \\ 2 & 0 & 10 & (9) & \text { homogeneous } & 65.32 \% & 11.14 \%\end{array}$

Observations: In tech level 1 , the pattern structure effect is remarkably strong, which is interesting as the level is not much different to the balanced scenario, where the effect is of minor importance. The other effects are slightly weaker than for the balanced case. In level 2, the SOM size and iterations effects take over again, and the overall effect sizes increase to a difference of about $10 \%$ between best and worst. Level 2 is the only scenario with several significant interactions, lead by two interactions of the iterations parameter. The iterationspattern structure effect means that low iterations/mixed plus high iterations/homogeneous is much worse than the two other combinations. This is probably due to the fact that large iteration numbers are not that important for homogeneous patterns than they are for mixed ones. But the overall interaction effect strengths are much lower than the main effect strengths, as in tech level 1 and 3. For tech level 3, the SOM size and iterations effects are already very dominant and their absolute values are very high, leading to a $20 \%$ relative difference between best and worst configurations.

The well trained large SOMs approximate the optimum nearly perfectly, whereas the smaller SOMs barely reach $80 \%$ 
of the optimal substitute objective function value. Interestingly, the random (untrained) SOMs also reach relatively high qualities, although they stay well below the trained SOMs. Training - in our context equivalent to applying an optimization-undoubtedly has an effect.

Discussion: Regarding to the sought good and robust parametrization of the learning process, we can state that the parameter effects are nearly never contradictory, only their strength varies. For the more demanding scenarios with more unit types, a larger SOM clearly is an advantage, accompanied by a larger number of training iterations. Applying only homogeneous training patterns is an advantage for the simpler scenarios, whereas it loses its importance for the more complex ones. Summarizing, one may always set the SOM size, iteration and unit numbers to high values, and train only with the homogeneous patterns. The low effect of the training patterns for tech level 3 could be interpreted as difficulty to produce meaningful mixtures with many unit types, too many of the randomly created patterns may be unreasonable and can thus not support the learning process. The comparison of the relative quality reached by different SOMs over the whole spectrum of 45 test cases for tech level 3 indicate that the large, trained SOM is clearly superior to its small and untrained counterparts, and shows little variance, making it very reliable. In some cases, the optimal defending group has not been attained. Here the EA based optimization may not be strong enough and may be developed further. However, the big picture is that the given answer is quite close to the optimal one.

To clarify what the SOM actually learns in terms of suggested defense unit groups, we additionally plot the highest rated unit type for each SOM node in three subsequent learning states for a well configured SOM on tech level 3 in fig. 10. Only few unit types remain after 1,000 iterations, and most nodes favor air units (ornithopters and airships). The domination of these air units for tech level 3 could have several reasons. Firstly, it may of course be intentional as the more advanced units shall be stronger. Adding to this, we completely neglect cost and speed and many other unit attributes in our substitute objective function, which may compensate for unbalanced attack strengths in the game. Secondly, the majority of unit types has only short-range attack skills, which reflects in the training patterns. Air units have the advantage that they cannot be attacked by short-range units. So, ensuring that skills and features are uniformly distributed in the training patterns might help. Another alternative would be to record group constellations from playing humans and to use this data as training input. Thirdly, incorporating cross validation into the training process could be helpful, too.

\section{CONCLUSIONS}

SOMs support a structured, content-addressable storage of information. They can be trained offline during the production of a game and remain static while playing. Training a SOM is a matter of few minutes even for the largest investigated configurations, and this time can probably even be reduced when training only with homogenous patterns, as our experiments indicate. These homogenous patterns are canonical, so that there is no specific information or experience to gather before starting the learning process. The suggested parametrization is robust and works well on all tested settings.

Even if different conditions (different unit types due to upgrades or new buildings) can occur within a played game, matching SOMs for them can be pre-produced, so that game AI just has to switch between them. This may be an important advantage for game producers who have to make sure that the game AI is stable and behaves as intended. SOMs also do not impose restrictions on how to use their information. So they could be combined with rule-based systems which still prevail in commercial games.

As shown in this work, it is simple and effective to enhance defending group selection (anti-grouping) in the game AI by means of SOMs. However, there are more possible uses, e.g. the tactical attack and strategic build decisions which may be supported by a similar approach, as outlined in the introduction. Furthermore, SOMs are easy to visualize, as shown in fig. 10, making them a handy supportive tool for game development issues like e.g. unit balancing, a road that shall be explored by us in the near future.

\section{Acknowledgements}

This work was kindly supported by the Deutsche Forschungsgemeinschaft $(D F G)$ and the Federal Ministry of Economics and Technology (BMWi). We thank the student project group 511 for developing the basics of this work.

\section{REFERENCES}

[1] H. Danielsiek, C. Eichhorn, T. Hein, E. Kurtić, G. Neugebauer, N. Piatkowski, J. Quadflieg, S. Schnelker, R. Stüer, A. Thom, and S. Wessing, "Final report of PG 511," Technische Universität Dortmund, Tech. Rep. Reihe CI 252/08, SFB 531, 2008.

[2] M. Figueroa, J. González, T. Fernández, F. Menéndez, and M. Caruncho, "Glest, a free 3d real time strategy game," 2007 , December 16, 2007. [Online]. Available: http://www.glest.org

[3] T. Kohonen, Self-Organizing Maps. Berlin: Springer, 2001.

[4] H.-G. Beyer and H.-P. Schwefel, "Evolution strategies - a comprehensive introduction," Natural Computing, vol. 1, no. 1, pp. 3-52, 2002.

[5] T. Kohonen, "Fast evolutionary learning with batch-type selforganizing maps," Neural Processing Letters, vol. 9, no. 2, pp. 153$162,1999$.

[6] S. J. Louis and J. McDonnell, "Learning with case-injected genetic algorithms." IEEE Trans. Evolutionary Computation, vol. 8, no. 4, pp. 316-328, 2004.

[7] S. J. Louis and C. Miles, "Case-injection improves response time for a real-time strategy game." in Proceedings of the 2005 IEEE Symposium on Computational Intelligence and Games (CIG05), G. Kendall and S. Lucas, Eds. IEEE, 2005.

[8] F. Schadd, S. Bakkes, and P. Spronck, "Opponent modeling in realtime strategy games," in 8th International Conference on Intelligent Games and Simulation (GAME-ON 2007), M. Roccetti, Ed., 2007, pp. 61-68.

[9] M. J. V. Ponsen, P. Spronck, H. Muñoz-Avila, and D. W. Aha, "Knowledge acquisition for adaptive game ai," Sci. Comput. Program., vol. 67, no. 1, pp. 59-75, 2007.

[10] M. Preuß, "Reporting on experiments in evolutionary computation," Technische Universität Dortmund, Tech. Rep. Reihe CI 221/07, SFB 531, 2007

[11] T. Bartz-Beielstein, Experimental Research in Evolutionary Computation - The New Experimentalism, ser. Natural Computing Series. Springer, Berlin, 2006. 